\title{
HOMOLOGY OF CLOSED GEODESICS IN CERTAIN RIEMANNIAN MANIFOLDS
}

\author{
ATSUSHI KATSUDA AND TOSHIKAZU SUNADA ${ }^{1}$
}

\begin{abstract}
It is shown, by using the trace formula of Selberg type, that every primitive, one-dimensional homology class of a negatively curved compact locally symmetric space contains infinitely many prime closed geodesics.
\end{abstract}

0. Let $M$ be a compact space form of a symmetric space of rank one. In this note, we prove that each homology class in $H_{1}(M, \mathbf{Z})$ contains infinitely many free homotopy classes of closed curves, that is, the mapping induced from the Hurewicz homomorphism $\left[\pi_{1}(M)\right] \rightarrow H_{1}(M, \mathbf{Z})$ is an $\infty$-to-one correspondence. One of the geometric consequences is that any primitive homology class contains infinitely many prime closed geodesics, since, as was shown by Hadamard, every nonnull homotopy class contains a closed geodesic which is automatically prime if the homology class is primitive. Here a homology class $\alpha$ is called primitive if $\alpha$ is not a nontrivial integral multiple of another homology class. If $\operatorname{dim} M=2$, then one can prove the much stronger assertion that every homology class contains infinitely many prime closed geodesics (see $\S 2$ ).

The following theorem, which can be shown by means of a number-theoretic argument applied to the $L$-functions associated to length spectrum of closed geodesics (see $[\mathbf{1}, \mathbf{4}]$ for proof), is somewhat related to the result.

THEOREM. Let $H$ be a subgroup of $H_{1}(M, \mathbf{Z})$ of finite index, and let $\alpha$ be a coset in $H_{1} / H$. If $M$ is negatively curved, then there exist infinitely many prime closed geodesics whose homology classes are in $\alpha$.

1. The proof relies heavily on the trace formula for the heat kernel function. We shall start with a general setting. Let $\pi: \tilde{M} \rightarrow M$ be the universal covering of a compact Riemannian manifold $M$. The fundamental group $\pi_{1}(M)$ acts on $\tilde{M}$ in the usual way. For brevity we write $\Gamma$ for $\pi_{1}(M)$. For an element $\gamma$ in $\Gamma$, we denote by $\Gamma_{\gamma}$ the centralizer of $\gamma$, and by $[\gamma]$ the conjugacy class of $\gamma$. The set of all conjugacy classes is denoted by $[\Gamma]$. Let $\rho: \Gamma \rightarrow U(N)$ be a unitary representation, and let $E_{\rho}$ be the flat vector bundle associated to $\rho$. We denote by $\Delta_{\rho}$ the Laplacian acting on the sections of $E_{\rho}$. The fundamental solution of the heat equation on $\tilde{M}$ will be denoted by $\tilde{k}(t ; \tilde{x}, \tilde{y})$. The following lemma is proved in the same way as the proof of the Selberg trace formula (see [6]).

LEMMA 1.

$$
\operatorname{tr}\left(e^{-t \Delta_{\rho}}\right)=\sum_{[\gamma] \in[\Gamma]} \operatorname{tr} \rho(\gamma) \int_{\tilde{M} / \Gamma_{\gamma}} \tilde{k}(t ; \tilde{x}, \gamma \tilde{x}) d \tilde{x} .
$$

Received by the editors January $22,1985$.

1980 Mathematics Subject Classification. Primary 53C35, 53C22.

${ }^{1}$ Supported by the Ishida Foundation. 
We set

$$
\begin{aligned}
f_{[\gamma]}(t) & =\int_{\tilde{M} / \Gamma_{\gamma}} \tilde{k}(t ; \tilde{x}, \gamma \tilde{x}) d \tilde{x}, \\
f_{\alpha}(t) & =\sum_{[\gamma] \in \alpha} f_{[\gamma]}(t),
\end{aligned}
$$

where $[\gamma] \in \alpha$ means that $\alpha$ is the image of $[\gamma]$ by the canonical mapping $[\Gamma] \rightarrow$ $\Gamma /[\Gamma, \Gamma]=H_{1}(M, \mathbf{Z})$.

LEMMA 2. $\varlimsup_{t \rightarrow \infty} t^{-1} \log f_{\alpha}(t)=0$.

PROOF. We denote by $\hat{H}_{1}$ the group of one-dimensional characters of $H_{1}(M, \mathbf{Z})$. Using the orthogonal relations of characters, we get

$$
f_{\alpha}(t)=\int_{\hat{H}_{1}} \chi\left(\alpha^{-1}\right) \operatorname{tr}\left(e^{-t \Delta_{\chi}}\right) d \chi
$$

where $d \chi$ is the normalized Haar measure on the compact group $\hat{H}_{1}$. Since $f_{\alpha}$ and $\operatorname{tr}\left(e^{-t \Delta_{x}}\right)$ are real valued, we find

$$
f_{\alpha}(t)=\int_{\hat{H}_{1}} \operatorname{Re} \chi\left(\alpha^{-1}\right) \operatorname{tr}\left(e^{-t \Delta_{\chi}}\right) d \chi
$$

Note that the first eigenvalue $\lambda_{1}(\chi)$ of the Laplacian $\Delta_{\chi}$ depends continuously on $\chi$, and that $\lambda_{1}(\chi)=0$ if and only if $\chi$ is the trivial character. Hence, $\lambda_{1}(\chi) \geq \mu$ for some positive $\mu$ on the compact set $K=\left\{\chi \in \hat{H}_{1}: \operatorname{Re} \chi\left(\alpha^{-1}\right) \leq 0\right\}$, and for each $\chi \in K$,

$$
\operatorname{tr}\left(e^{-t \Delta_{\chi}}\right)=e^{-\lambda_{1}(\chi) t}+e^{-\lambda_{2}(\chi) t}+\cdots=e^{-\mu t} C \text { for } t \gg 0,
$$

where the constant $C$ can be chosen independently from $\chi \in K$.

We now suppose that

$$
\varlimsup_{t \rightarrow \infty} t^{-1} \log f_{\alpha}(t)<\lambda<0 .
$$

This implies that $f_{\alpha}(t)<e^{-t \lambda}$ for $t \gg 0$. If we set $\theta=\min (\mu, \lambda)$, then we have

$$
\int_{\operatorname{Re} \chi\left(\alpha^{-1}\right)>0} \operatorname{Re} \chi\left(\alpha^{-1}\right) \operatorname{tr}\left(e^{-t \Delta_{\chi}}\right) d \chi \leq C e^{-t \theta} .
$$

Take a positive $\varepsilon$ less than $\theta$, and let $U$ be a neighborhood of the trivial character such that for $\chi \in U, \operatorname{Re} \chi\left(\alpha^{-1}\right) \geq c_{0}>0, \lambda_{1}(\chi) \leq \varepsilon$. We then find that

$$
\begin{aligned}
& \int_{\operatorname{Re} \chi\left(\alpha^{-1}\right)>0} \operatorname{Re} \chi\left(\alpha^{-1}\right) \operatorname{tr}\left(e^{-t \Delta_{\chi}}\right) d \chi \\
& \quad \geq \int_{U} \operatorname{Re} \chi\left(\alpha^{-1}\right) \operatorname{tr}\left(e^{-t \Delta_{\chi}}\right) d \chi \geq c_{0} e^{-t \varepsilon} \int_{U} 1 d \chi
\end{aligned}
$$

This is a contradiction.

LEMMA 3. $\overline{\lim }_{t \rightarrow \infty} t^{-1} \log f_{[e]}(t) \leq-\lambda_{0}(\tilde{M})$, where $\lambda_{0}(\tilde{M})$ is the lowest bound of the spectrum of the Laplacian $\Delta_{\tilde{M}}$ on $\tilde{M}$.

PROOF. This comes from the spectral representation of $\tilde{k}(t ; \tilde{x}, \tilde{y})$ and the equality

$$
f_{[e]}(t)=\int_{M} \tilde{k}(t ; \tilde{x}, \tilde{x}) d \tilde{x}
$$


In fact one easily has

$$
\varlimsup_{t \rightarrow \infty} t^{-1} \log \tilde{k}(t ; \tilde{x}, \tilde{y}) \leq-\lambda_{0}(\tilde{M})
$$

LEMMA 4. If $M$ is a locally symmetric space of negative curvature, then, for $\gamma \neq e$,

$$
\lim _{t \rightarrow \infty} t^{-1} \log f_{[\gamma]}(t)=-\lambda_{0}(\tilde{M})
$$

PROOF. The assertion is an immediate consequence of the trace formula established by R. Gangolli [2]:

$$
f_{[\gamma]}(t)=(4 \pi t)^{-1 / 2} e^{-\lambda_{0}(\tilde{M}) t} l_{[\gamma]}^{\prime}\left|\operatorname{det}\left(P_{[\gamma]}-I\right)\right|^{-1 / 2} \exp \left(-l_{[\gamma]}^{2} / 4 t\right),
$$

where $l_{[\gamma]}$ is the length of a closed geodesic $c$ with the homotopy class $[\gamma], l_{[\gamma]}^{\prime}$ is the length of the prime geodesic whose image coincides with $c$, and $P_{[\gamma]}$ is the linearized Poincaré mapping associated with $c$.

PROOF OF MAIN RESULT. If there exist only finitely many $[\gamma]$ with $[\gamma] \in \alpha$, then the above lemma implies

$$
\varlimsup_{t \rightarrow \infty} t^{-1} \log f_{\alpha}(t)<0,
$$

which contradicts Lemma 2.

REMARK. The trace formula in Lemma 1 can be connected with a summation formula for Wiener integrals on path spaces (see [6]).

2. If $\operatorname{dim} M=2$ and the genus of $M(=g)$ is greater than one, then $\pi_{1}(M)$ is isomorphic to the group generated by $2 g$ elements $A_{1}, \ldots, A_{g}, B_{1}, \ldots, B_{g}$ with the single relation $\prod_{i=1}^{h} A_{i} B_{i} A_{i}^{-1} B_{i}^{-1}=1$. This, in particular, implies that there exists a surjective homomorphism from $\pi_{1}(M)$ to the free group of rank $g$. We shall prove the following general theorem.

THEOREM. Let $M$ be a compact Riemannian manifold. If there exists a surjective homomorphism of $\pi_{1}(M)$ onto a nonabelian free group, then for any $\alpha \in$ $H_{1}(M, \mathbf{Z})$, there exist infinitely many prime geodesics in $\alpha$.

PROOF. From the assumption, one can make a surjective homomorphism onto the free group of rank two: $\pi: \pi_{1}(M) \rightarrow F_{2}=\left\langle a_{1}, a_{2}\right\rangle$. Take an element $\gamma$ in $\pi_{1}(M)$ whose image by the Hurewicz homomorphism is $\alpha$, and express $\pi(\gamma)$ by a reduced word $\pi(\gamma)=x_{1} \cdots x_{h}$, where $x_{i}=a_{1}^{\varepsilon}$ or $a_{2}^{\varepsilon}(\varepsilon= \pm 1)$. We set

$$
\begin{aligned}
& a(m, n)=x_{1} \cdots x_{h} a_{1}^{m} a_{2}^{n} a_{1}^{-m} a_{2}^{-n}, \\
& \gamma(m, n)=\gamma \gamma_{1}^{m} \gamma_{2}^{n} \gamma_{1}^{-m} \gamma_{2}^{-n}
\end{aligned}
$$

where $\pi\left(\gamma_{i}\right)=a_{i}(i=1,2)$. Note that $\gamma(m, n)$ is homologous to $\gamma$ in $H_{1}(M, \mathbf{Z})$ for any $(m, n)$. Without loss of generality, one may assume that the word $a(m, n)$ is reduced and cyclically reduced (if necessary, we take $a_{1}^{-1}$ and $a_{2}^{-1}$ instead of $a_{1}$ and $\left.a_{2}\right)$. See $[\mathbf{3}]$ for terminology.

We show that (i) $\gamma(m, n)$ is not a nontrivial power of another element if $n>h$, and (ii) $\gamma(m, n)$ is not conjugate to $\gamma\left(m^{\prime}, n^{\prime}\right)$ for $(m, n) \neq\left(m^{\prime}, n^{\prime}\right)$. For this, it is enough to prove the same assertion for $a(m, n)$.

Suppose that $a(m, n)=\left(y_{1} \cdots y_{k}\right)^{r}(r \geq 2)$, where $y_{1} \cdots y_{k}$ is a reduced word. Since $a(m, n)$ is cyclically reduced, so is $y_{1} \cdots y_{k}$, and

$$
\left(y_{1} \cdots y_{k}\right)^{r}=y_{1} \cdots y_{k} y_{1} \cdots y_{k} \cdots y_{1} \cdots y_{k}
$$


is reduced. This implies that the word $y_{1} \cdots y_{k}$ has the symbol $a_{2}^{-n}$ as the last $n$ symbols, so that $\left(y_{1} \cdots y_{k}\right)^{r}$ has at least $r n$ symbols $a_{2}^{-1}$. On the other hand, $a(m, n)$ has at most $h+n$ symbols $a_{2}^{-1}$. This is a contradiction.

For (ii), we use [3, Theorem 3.2, p. 36], which asserts that if $a(m, n)$ is conjugate to $a\left(m^{\prime}, n^{\prime}\right)$, then $a(m, n)$ is a cyclic permutation of $a\left(m^{\prime}, n^{\prime}\right)$. Since $(m, n) \neq$ $\left(m^{\prime}, n^{\prime}\right)$, the numbers of the symbols $a_{1}$ or $a_{2}$ in the words $a(m, n)$ and $a\left(m^{\prime}, n^{\prime}\right)$ are different. This implies (ii).

\section{REFERENCES}

1. T. Adachi and T. Sunada, L-functions of pro-finite graphs and dynamical systems, preprint, Nagoya, Univ., 1984.

2. R. Gangolli, Zeta functions of Selberg's type for compact space forms of symmetric spaces of rank one, Illinois J. Math. 21 (1977), 1-42.

3. W. Magnus, A. Karras and D. Solitar, Combinatorial group theory, Interscience, New York, 1966.

4. W. Parry and M. Pollicott, The Chebotarev theorem for Galois coverings of axiom A flows, preprint, Warwick Univ., 1984.

5. A. Selberg, Harmonic analysis and discontinuous groups in weakly symmetric spaces with applications to Dirichlet series, J. Indian Math. Soc. 20 (1956), 47-87.

6. T. Sunada, Trace formulas, Wiener integrals and asymptotics, Proc. Spectra Riemannian Manifolds, Kaigai Publ., Tokyo, 1983, pp. 103-113.

7. __ Riemannian coverings and isospectral manifolds, Ann. of Math. 121 (1985), 169186.

DEPARTMENT OF MATHEMATICS, NAGOYA UNIVERSity, NAGOYA 464, JAPAN 